BACTERIAL DEVELOPMENT

\title{
Racing to decide
}

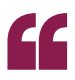

the sporulation

versus

competence

choice is

dependent on

a molecular

race between

the two

programmes
Cells that can differentiate towards one fate or another occur across species. However, understanding how an individual cell 'chooses' its fate is challenging; cross-regulation between competing programmes has been suggested as a mechanism, but recent work in bacteria shows that fate can depend on which programme wins a molecular race.

In conditions of stress, most Bacillus subtilis cells form spores, but a small percentage become competent (that is, they are able to take up DNA). Sporulation and competence are mutually exclusive fates, so Kuchina et al. used this developmental choice as a model to examine decision making. They were able to track the progress of these two molecular programmes in single cells over time by using fluorescent reporters coupled to the promoters of different genes that are known to be involved in regulating sporulation or competence programmes.

The authors found that the probability of initiating competence is independent of the progression of the sporulation programme. Furthermore, they found that genes that were previously shown to crossregulate these pathways act only after the decision point. Therefore, the sporulation and competence programmes do not cross-regulate each other before the decision point is reached. Presumably, cross-regulation after the decision ensures the correct execution of the chosen programme.

Kuchina et al. also observed that some cells that had almost reached the point of commitment to spore formation did initiate the competence programme by expressing the competence master regulator ComK. However, these cells always proceeded to form spores; there was insufficient time for the competence programme to run its course before spores formed. Therefore, the authors proposed that the sporulation versus competence choice is dependent on a molecular race between the two programmes. They tested this hypothesis by expressing com $K$ from promoters that normally drive gene expression at particular times during the sporulation process. Their results showed that altering the timing of $c o m K$ expression relative to the sporulation programme influenced the cell fate decision, confirming that timing controls fate.

A race is a simple system for decision making, and further single-cell studies should help to reveal the extent to which this mechanism occurs in other developmental contexts.

Mary Muers, Acting Chief Editor, Nature Reviews Genetics This article originally appeared in Nature Rev. Genet. (doi:10.1038/nrg3158).

ORIGINAL RESEARCH PAPER Kuchina, A. et al.

Temporal competition between differentiation programs determines cell fate choice. Mol. Sys. Biol. 7, 557 (2011)

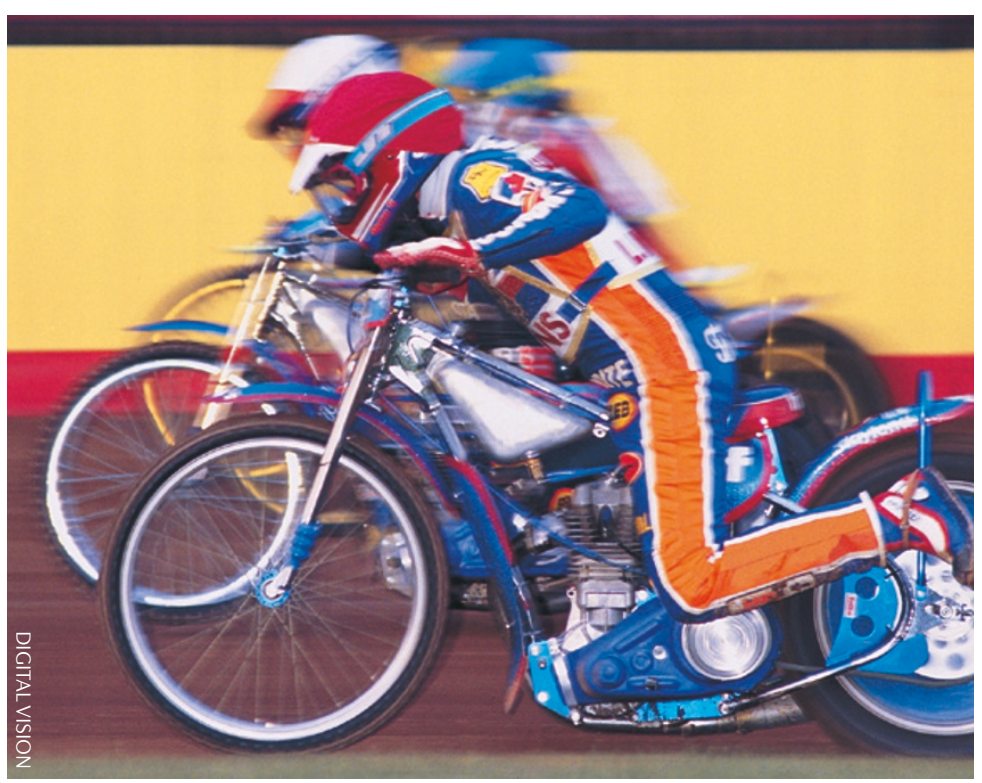

